\title{
Effect of Concurrent Chemoradiation for Advanced Head and Neck Malignancy in a Tertiary Care Centre, Kerala
}

\author{
Ravisankar Thommanparambil Raveendran ${ }^{1}$, Shehna Abdul Khader ${ }^{2}$, Ajith Kumar Vilasini \\ Raghavan ${ }^{3}$, Jayaraman Madambath Balan4, Krishnannair Lalithamma Jayakumar ${ }^{5}$ \\ 1, 2, 3, 4 Department of Radiotherapy, Government Medical College, Thrissur, Kerala, India, \\ ${ }^{5}$ Department of Oncology, Sreemookambika Institute of Medical Science, Kulasekharam, \\ KK District. Tamil Nadu, India.
}

\section{ABSTRACT}

\section{BACKGROUND}

Concurrent chemotherapy is a well-established treatment modality for locally advanced head and neck cancer. The concept of concurrent chemotherapy and radiation was introduced in an attempt to improve the local control and possibly influence the survival because of the high rate of local and distant failures observed with the combination of surgery and postoperative radiation. The relevance of this study was to assess the efficacy of our treatment and patience compliance and also study the effect in patients treated with cisplatin based concurrent chemo radiotherapy in advanced head and neck cancer.

\section{METHODS}

The prospective study was conducted in the Department of Radiotherapy, Government Medical college, Thrissur, Kerala comprising the newly diagnosed patients with locally advanced head \& neck cancers over one year. Conventional radiotherapy with a dose of 66 Gy in 33 fractions over 6.5 weeks was given concurrently with Inj cisplatin $100 \mathrm{mg} / 2$ IV every 3 weeks and periodically followed up for one year.

\section{RESULTS}

This study revealed that complete response rate was higher in 61 - 70 year age group compared to lower age groups. Complete response cases were slightly higher in T1 disease compared to higher stages. Regarding nodal status, complete response and DFS were more in N0 tumours and worst in N3 tumours. It was found that complete response rates were slightly higher in stage III than stage IV. Comparing the grade of the tumour, complete response cases were slightly higher in WD and MD compared to PD. Complete response rate and disease free survival (DFS) were slightly higher in cases who had more than two chemotherapy cycles compared to one cycle.

\section{CONCLUSIONS}

Concurrent chemo radiation was not well tolerated in our study group. Only $23.5 \%$ patients were able to complete the planned treatment. The positive side was that complete response was found in about $79.4 \%$ of study patients \& DFS at one year was $80 \%$.

\section{KEY WORDS}

Concurrent Chemo Radiation, Head and Neck Cancer, Cisplatin
Corresponding Author: Dr. Ajith Kumar V.R., Department of Radiotherapy, Government Medical College, Thrissur, Kerala, India.

E-mail: devu666@yahoo.co.in

DOI: $10.14260 / j e m d s / 2021 / 428$

How to Cite This Article: Raveendran RT, Khader SA, Raghavan AKV, et al. Effect of concurrent chemoradiation for advanced head and neck malignancy in a tertiary care centre, Kerala. J Evolution Med Dent Sci 2021;10(28):2094-2098, DOI: $10.14260 / \mathrm{jemds} / 2021 / 428$

Submission 28-01-2021,

Peer Review 11-05-2021,

Acceptance 19-05-2021,

Published 12-07-2021.

Copyright (C) 2021 Ravisankar Thommanparambil Raveendran et al. This is an open access article distributed under Creative Commons Attribution License [Attribution 4.0 International (CC BY 4.0)] 


\section{BACKGROUND}

Head and neck cancers are the most common cancers in developing countries. ${ }^{1}$ Oral cancers are predominant forms of head and neck squamous cell cancer (HNSCC) in India, Pakistan, and other Southeast Asian countries; oropharyngeal and tongue cancers are common in the Western world. ${ }^{2}$ Cigarette smoking and alcohol consumption are the main reasons for HNSCC in the Western population, whereas the use of smokeless tobacco and areca nut is the most common cause of HNSCC in Southeast Asia. ${ }^{3,4}$

Head and neck cancer is an area of great importance to the researchers and oncologists because of the psychological and physical morbidity it produces and also causes significant burden on the family and society. For the management of head and neck SCC, either surgery or radiotherapy can be used as a primary treatment.

Chemotherapy has also proven to have a role when concurrently administered with radiotherapy 5 as a general rule, and in an effort to minimize the side effects of multiple treatment modalities, a monotherapy approach is preferred to treat head and neck SCC, as long as it does not negatively impact loco regional control or survival.

For early-stage cancers, a single modality treatment (surgery or radiotherapy) is usually deemed to be sufficient to achieve cure, and as a consequence, one of those two options is employed. However, for advanced stage cancers, monotherapy is insufficient to control the disease.

Thus, a combined approach of surgery followed by adjuvant radiation with or without concurrent chemotherapy or primary chemo radiotherapy without surgery is used. ${ }^{6}$ surgical resection of many head and neck tumours can result in significant functional impairment due to complexity in anatomy. Traditional open surgical approaches can result in severe dysphagia and aspiration, which may result in patients depending on tracheostomy and / or gastrostomy tube.

Consequently, in scenarios where similar oncologic outcomes between surgery and radiotherapy can be achieved, the latter approach has been traditionally preferred with the idea of organ preservation as it is thought that radiation treatment might cause less morbidity.

Concurrent chemotherapy is a well-established treatment modality for locally advanced head and neck cancer. The concept of concurrent chemotherapy and radiation was introduced in an attempt to improve the local control and possibly influence the survival because of the high rate of local and distant failures observed with the combination of surgery and postoperative radiation. In our department there is only a cobalt 60 machine with 2 dimensional planning for treating a large no of patients daily.

The relevance of this study was to assess the efficacy of our treatment and patience compliance.

In our department around 4000 new cancers are registered annually, out of which $16 \%$ constitute head and neck malignancies. This study intended to analyse the effects of concomitant chemo radiation (CRT) in locally advanced squamous cell carcinoma of oral cavity, oropharynx, hypopharynx and larynx (STAGE III \& IVA)

\section{METHODS}

This prospective study was conducted in the Department of Radiotherapy, Government Medical College, Thrissur, Kerala comprising the newly diagnosed patients with locally advanced stage III and IV a squamous cell carcinoma (based on AJCC 8th edition) of oral cavity, oropharynx, hypopharynx and larynx for a period of one year from April 2018 to August 2019. The patients included had histologic or cytologic confirmation of squamous cell carcinoma with age less than 70 years and Eastern cooperative oncology group (ECOG) performance status of 0,1 . Nasopharyngeal carcinomas, patients treated with Neoadjuvant chemotherapy, initial surgical treatment excluding diagnostic biopsy of the primary site or neck disease and patients with simultaneous primaries were excluded. Ethical committee clearance was obtained from Communication of Decision of the Institutional Ethics Committee (IEC) / Institutional Review Board (IRB) dated 17 / 11 / 2017 (Order No: B6 - 8772 / 2016 / MCTCR)

\section{Sample Size Calculation}

Sample size was calculated using the formula $n=2 E /(2-$ $\left.\pi_{1}-\pi_{2}\right)$

WhereE $=\left[\frac{(H R+1)^{2}\left(Z_{1-\alpha / 2}+Z_{1-\beta}\right)^{2}}{(H R-1)^{2}}\right]$ $n=m=(N / 2) H R=\ln (\pi 2) / \ln (\pi 1)$

$\pi 1=$ proportion of survival in treatment group $=43$

A study conducted by TATA memorial hospital5,7 by Tejpal Gupta et al. the disease free survival was $43 \%$, $\pi 2=$ proportion of survival in control group $\alpha=$ significance level $=5 \%$ and $1-\beta=$ power $=99 \%$

So final sample size was 25 .

\section{Sampling Method \& Selection of Study Participants}

Consecutive patients fulfilling the inclusion criteria and willing to give consent, were recruited for the study till the desired sample size was met. Conventional radiotherapy was planned after appropriate immobilization using thermoplastic mask. All patients were irradiated with megavoltage external beam radiotherapy using telecobalt machine with conventional fractionation (2Gy per fraction, one fraction per day, 5 days per week). The gross tumour volume was treated to a dose of 66 Gy in 33 fractions over 6.5 weeks. Areas of potential microscopic disease were treated to a dose of 50 Gy in 20 fractions on field over 4 weeks. The radiation portals were dictated by primary site and disease stage. Field size reduction was done after 44 Gy in 22 fractions considering the spinal cord tolerance. Concurrent chemotherapy with Inj cisplatin was given on $100 \mathrm{mg} / \mathrm{m}^{2}$ IV on Day 1, Day 22 and Day 43. General nutritional status \& change in performance scores were monitored every week. All treatment times were documented on the treatment chart.

Study participants were followed up for every 3 months until the next 1 year. During the follow up visits clinical examination was done for detection of loco - regional disease. Complete clinical response is defined as the complete disappearance of all detectable tumour. Disease free survival 
period starts from the time of completion of chemo radiation up to the time of clinical / radiological recurrence or one year follow up period whichever is earlier. The disease status of patients who had completed the planned course of therapy, but not follow up were updated by telephonic interview. Nonresponding patients were considered lost to follow up and censored for statistical consideration.

\section{Statistical Analysis}

Descriptive analysis and logical regression was performed by nMaster 2.0 software. P value was calculated and the value < 0.05 was considered significant.

\section{RESULTS}

Among 34 patients ( 30 males \& 4 females) $23.5 \%$ of the cases belonged to the age group 41 - 50 years and $47.1 \%$ of the cases belonged to the group 51 - 60 years. Around $29.4 \%$ cases with age 61 - 70 years were also included. The average age was 56.32 years with standard deviation 7.010. The minimum and maximum age were 42 and 67 years respectively. Majority $(79.4 \%)$ of the cases had the habit of both smoking and alcoholism, $8.8 \%$ had only smoking and $8.8 \%$ pan chewing. In this study, $29.4 \%$ of the cases were having oral cavity primary and $26.5 \%$ were having oropharyngeal cancer. Regarding the primary site of disease $23.5 \%$ was in the larynx and $20.6 \%$ of the cases were in hypopharynx. It was observed that most of the patients i.e. $35.3 \%$ were staged T2, $29.4 \%$ T3 and $26.5 \%$ T4. Only $8.8 \%$ patients belonged to T1 stage. Fifty percent of patients in the study presented with $\mathrm{N} 2$ nodal status, $29.4 \%$ with N1, $17.6 \%$ with N0, and only $2.9 \%$ patients had N3 nodal status. Fifty percent of the participants had moderately differentiated (MD) tumours, $44.1 \%$ with well differentiated tumours (WD), and only $5.9 \%$ patients had poorly differentiated tumours.

Only $29.4 \%$ of patients completed full 3 cycles of chemotherapy, majority of the patients (55.9\%) had 2 cycles and rest of the patients (14.7\%) were able to undergo only 1 cycle of chemotherapy. Among 34 cases included in the study, $64.7 \%$ of the cases survived the follow up period without any evidence of recurrence and 4 patients i.e., $14.7 \%$ had recurrence. Seventy-nine percent had complete clinical response and the rest had only partial response.

\section{Response According to Patient Characteristics}

This study revealed that complete response rate was higher in 61 - 70 year age group (100\%) and $51-60$ years $(81.3 \%)$ compared with lower age of $41-50$ years $(50.0 \%)$ with P value less than the significance level of 0.05 ; and $P$ value was almost same in both males (80.0\%) and females (75.0\%). The study showed that complete response cases were slightly higher (P value 0.725$)$ - in T1 disease $(100.0 \%)$ compared to T2 (75.0\%), T3 (70.0\%) and T4 (88.9\%) diseases.

Regarding nodal status, complete response cases were more in N0 tumours (100\%) and worst in N3 tumours (0\%) $P$ value was 0.790 compared to $\mathrm{N} 1(80.0 \%)$ and $\mathrm{N} 2$ (82.4\%). It was found that complete response rates were slightly higher in stage III (80.0 \%) than stage IV (78.9\%). Taking in to account of the grade of the tumour, complete response cases were slightly higher in WD (80.0\%) and MD (82.4\%) compared to PD (50.0\%) (P value 0.454$)$. In this study, it is found that there was statistically significant relationship between response and number of chemotherapies ( $\mathrm{P}$ value 0.003). Complete response rate was slightly higher in cases who had two chemotherapies $(84.2 \%)$ and three chemotherapies $(80.0 \%)$ compared to the cases with one chemotherapy (60.0\%). (Figure 1 )

\section{Relationship of DFS with Various Factors}

Considering the age of the patient, there was no statistically significant relationship between disease free survival (DFS) and age. It was seen that disease free survival was almost same in cases with age 41 - 50 years $(75.0 \%), 51$ - 60 years $(76.9 \%)$ and $61-70$ years $(90.0 \%)$. Observing the relationship between disease free survival and $\mathrm{T}$ - Stage, the $\mathrm{P}$ - value was not significant. Disease free survival was slightly lower (P value 0.573$)$ in T1 (66.7 \%) compared to T2 (88.9\%), T3 (71.4 $\%$ ) and T4 (87.5 \%). (Figure 2)

Regarding the nodal status, there was no relationship between disease free survival and $\mathrm{N}$ - stage. The study revealed that disease free survival was slightly higher in $\mathrm{N} 0$ $(1.00 \%)$ compared to $\mathrm{N} 1(75.0 \%)$ and $\mathrm{N} 2$ (78.6\%) (P value 0.856 ) (Figure 3). In the study DFS was almost same in stage III $(83.3 \%)$ and stage IV (80.0\%) and it was higher in WD (91.7\%) compared to MD (71.4\%) (P value 0.990$)$.

Statistically relating the number of chemotherapies and DFS there was a significant relationship between both (P values 0.003 ). The table reveals that disease free survival is significantly higher in cases with three chemotherapies $(100.0$ $\%)$ and two chemotherapies (87.5\%) compared to the cases with one chemotherapy $(0.0 \%)$. (Table 1$)$

\begin{tabular}{|ccccc|}
\hline $\begin{array}{c}\text { Number of } \\
\text { Chemo }\end{array}$ & \multicolumn{2}{c|}{ Recurrence } & Total & $\begin{array}{c}\text { P - } \\
\text { Value }\end{array}$ \\
One & $0(0.0 \%)$ & $3(100.0 \%)$ & 3 & \\
Two & $14(87.5 \%)$ & $2(12.5 \%)$ & 16 & 0.003 \\
Three & $8(100.0 \%)$ & $0(0.0 \%)$ & 8 & \\
Total & $\mathbf{2 2}(\mathbf{8 1 . 5} \%)$ & $\mathbf{5}(\mathbf{1 8 . 5} \%)$ & $\mathbf{2 7}$ & \\
\hline Table 1. Relationship between Disease Free Survival \\
and Number of Chemotherapy
\end{tabular}

\begin{tabular}{|ccccc|}
\hline Trial & Year & No of Patients & DFS (1 Year) & Complete Response \\
\hline Mach NC & 2009 & 17346 & $63 \%$ & $80 \%$ \\
\hline Intergroup & 2003 & 295 & $77 \%$ & $40.20 \%$ \\
\hline RTOG 91 11 & 2003 & 520 & $75 \%$ & $83 \%$ \\
\hline Tejpal Gupta et al. & 2009 & 264 & $75 \%$ & - \\
\hline Present study & 2019 & $34 \%$ & $79.4 \%$ \\
\hline
\end{tabular}



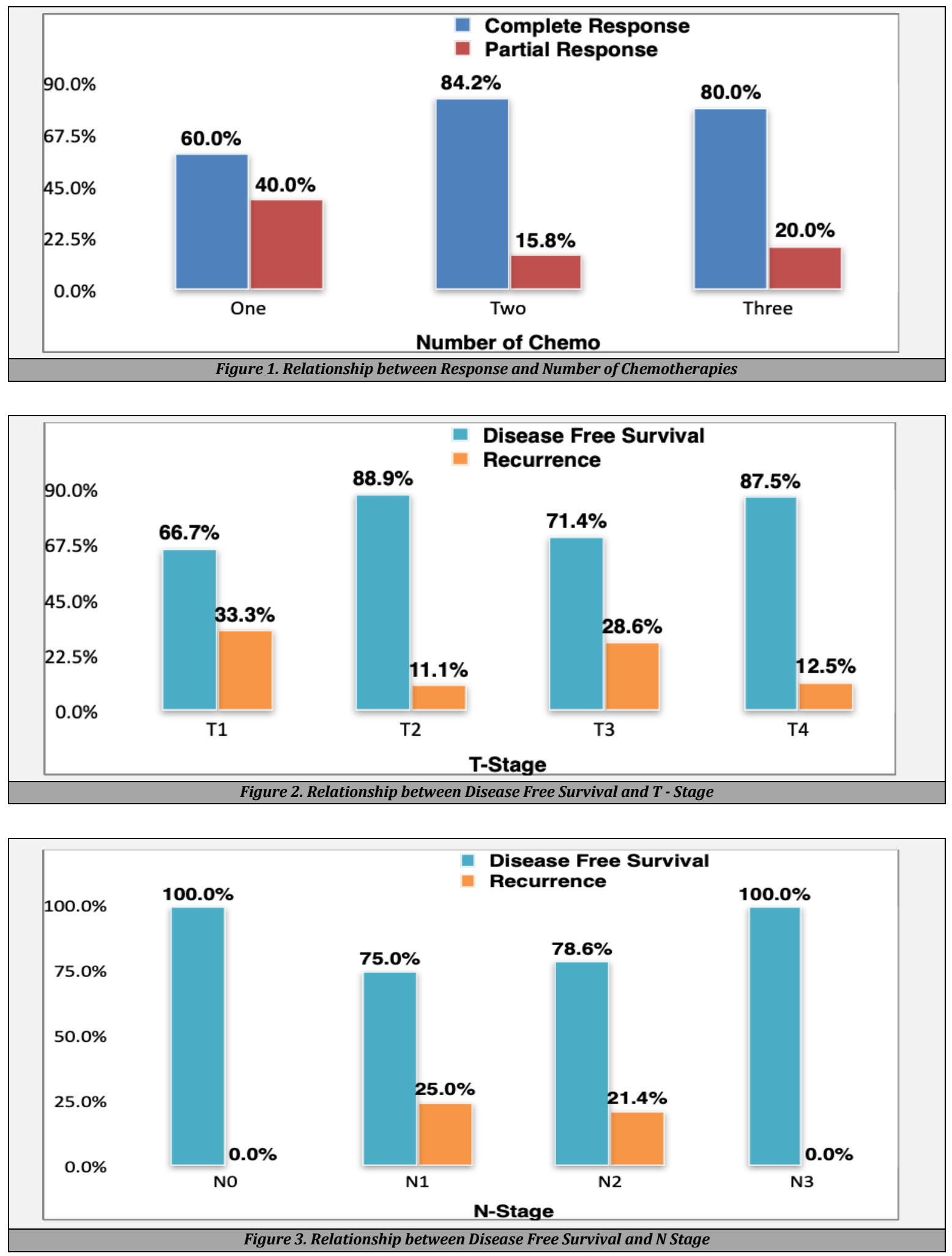

\section{DISCUSSION}

In the study most of the patients were of age group $51-60$ years, with a mean age of 56 years. Majority of patients were males (88.2\%) as seen in most of the studies. Following treatment about $80 \%$ patients had complete response and 20 $\%$ patients had partial response. This was comparable to the result obtained in the RTOG 91 - 11 trial (78\%) 37 and better than intergroup trial (40.2\%). Males had better complete response than females ( $80 \%$ VS $75 \%$ ); but $\mathrm{P}$ value 0.990 .
Disease free survival was also found to be better in males $(83.3$ \% VS $66.7 \%$; but P value 0.474 . Majority of the patients in our study were addicted to both smoking and alcohol (74.9\%) which had a synergistic effect on the occurrence of squamous cell carcinoma of head and neck. The risk ratio increased more in a multiplicative than in an additive manner. ${ }^{8}$

Anatomically most of the patients had oral cavity cancer (29.4\%), followed by oropharyngeal cancer (26.5\%) and least with hypopharynx carcinomas $(20.6 \%)$ as seen in most of the epidemiological studies.9,10,11 It was found that complete response cases were higher in oral cavity (85.7\%), larynx 
(80.0\%), hypopharynx (77.8\%) and oropharynx (75.0\%). Twelve patients (35.3\% had T2 status, 10 (29.3\%) had T3, 9 (26.5\%) had T4 and 3 (8.8\%) had T1. Complete response was seen in T1 (100\%), T2 (75\%), T3 (70\%), T4 (88\%) (P value - 0.725). The higher complete response rate found may be because of less number of $\mathrm{T} 4$ disease cases recruited for the study. DFS at 12 months was best seen in T2 tumours - $88.9 \%$ ( $P$ value 0.573 ). Since the P-value was greater than the significance level 0.05 there was no statistically significant relationship between disease free survival and $\mathrm{T}$ - stage in the study. In the present study 15 patients (44.1\%) belonged to stage III and 19 patients (55.9\%) belonged to stage IV. Complete response was slightly higher in stage 3 (80.0\%) compared to stage 4 (78.9\%). Disease free survival was also slightly better in stage III patients compared to stage IV (83.3 $\%$ VS $80.0 \%$ ) (P value 0.990$)$. Only 8 patients (23.5 \%) out of 34 completed 3 cycles of concomitant cisplatin-based chemotherapy. Most of the patients could not complete 3 courses due to mucositis and haematological toxicity of concurrent chemo radiation. Complete response was higher in patients who underwent more than 2 cycles of chemotherapy compared to those who underwent only single cycle $(84.2 \%$ VS $60 \%$ ) (P value 0.483 ). All of the patients were able to complete the planned radiation without breaks. Disease free survival was significantly higher in cases with three chemotherapies (100.0\%) and two chemotherapies (87.5\%) compared to the cases with one chemotherapy $(0.0 \%)$. ( $\mathrm{P}$ value 0.003 ) which was comparable to the results in RTOG 0129 trial. ${ }^{12}$

There was no statistically significant relationship between disease free survival and grade of the tumour ( $P$ value 0.454). It is clear that that disease free survival was higher in well differentiated tumours $(91.7 \%)$ compared to moderately differentiated tumours (71.4\%) (P value 0.454 )

To conclude, the overall disease-free survival was $80 \%$ at one year, this finding correlated with $75 \%$ at Tejpal Gupta et al. ${ }^{13} 63 \%$ at 1 year in MACH - NC, $77 \%$ at 1 year in intergroup trial, $75 \%$ at 1 year in RTOG $91-11.15$ (Table 2)

\section{CONCLUSIONS}

Concurrent chemoradiation is an established entity for the treatment of locally advanced head and neck malignancy. Concurrent chemo radiation with 3 weekly cisplatin was not well tolerated in our study group. Only $23.5 \%$ patients were able to complete the planned treatment with 3 cycles of chemotherapy concurrently with radiotherapy. The bright side was that complete response was found in about $79.4 \%$ of the study patients in one year. Disease free survival at one year was $80 \%$ which is very well in comparison with other major trials.

Data sharing statement provided by the authors is available with the full text of this article at jemds.com.

Financial or other competing interests: None.

Disclosure forms provided by the authors are available with the full text of this article at jemds.com.

\section{REFERENCES}

[1] WHO 2008. The global burden of disease: 2004 update. www.who.int/evidence/bod (accessed January 27, 2014).

[2] Bhurgri Y, Bhurgri A, Usman A, et al. Epidemiological review of head and neck cancers in Karachi. Asian Pac J Cancer Prev 2006;7(2):195-200.

[3] Graham S. Dentition, diet, tobacco and alcohol in the epidemiology of oral cancer. J Natl Cancer Inst 1977;59(6):1611-8.

[4] Dayal PK, Mani NJ, Bhargava K. Prevalence of oral cancer and precancerous lesions in pan/supari chewers. Indian J Public Health 1978;22(3):234-45.

[5] Pignon J, Le Maître A, Maillard E, et al. Meta-analysis of chemotherapy in head and neck cancer (MACH-NC): an update on 93 randomised trials and 17,346 patients. Radiother Oncol 2009;92(1):4-14.

[6] Soo KC, Tan EH, Wee J, et al. Surgery and adjuvant radiotherapy vs concurrent chemoradiotherapy in stage III/IV nonmetastatic squamous cell head and neck cancer: a randomised comparison. Br J Cancer 2005;93(3):27986.

[7] David AJ, Li Y, George AL, et al. An intergroup phase III comparison of standard radiation therapy and two schedules of concurrent chemoradiotherapy in patients with unrespectable squamous cell head and neck cancer. J Clin Oncol 2003;21(1):92-8.

[8] Maier H, Dietz A, Gewelke U, et al. Tobacco and alcohol and the risk of head and neck cancer. Clin Investig 1992;70(3-4):320-7.

[9] Sankaranarayanan R, Masuyer E, Swaminathan R, et al. Head and neck cancer: a global perspective on epidemiology and prognosis. Anticancer Res 1998;18(6B):4779-86.

[10] ICMR. Incidence, distribution, trends in incidence rates and projections of burden of cancer. In: Three-year report of population based cancer registries 2012-2014. Bengaluru, India: Indian Council of Medical Research 2016.

[11] ICMR. An assessment of the burden and care of cancer patients. In: Consolidated report of hospital based cancer registries 2012-2014. Bengaluru, India: Indian Council of Medical Research 2016.

[12] Ang K, Zhang Q, Wheeler RH, et al. A phase III trial (RTOG 0129) of two radiation-cisplatin regimens for head and neck carcinomas (HNC): impact of radiation and cisplatin intensity on outcome. Journal of Clinical Oncology 2010;28(Suppl 15):5507.

[13] Gupta T, Chopra S, Agarwal JP, et al. Squamous cell carcinoma of the hypopharynx: single-institution outcome analysis of a large cohort of patients treated with primary non-surgical approaches. Acta Oncol 2009;48(4):541-8.

[14] Forastiere AA, Zhang Q, Weber RS, et al. Long-term results of RTOG 91-11: a comparison of three nonsurgical treatment strategies to preserve the larynx in patients with locally advanced larynx cancer. J Clin Oncol 2013;31(7):845-52. 